\title{
Restore your confidence
}

Give your patients a reason to smile with the TLX implant system from Straumann.

Restorative procedures can sometimes be stressful for the clinician and the patient - especially when problems arise during the healing process. However, the TLX implant system from Straumann brings you state of the art connection technology. Designed to optimise the restorative workflow, TorcFit connection properly
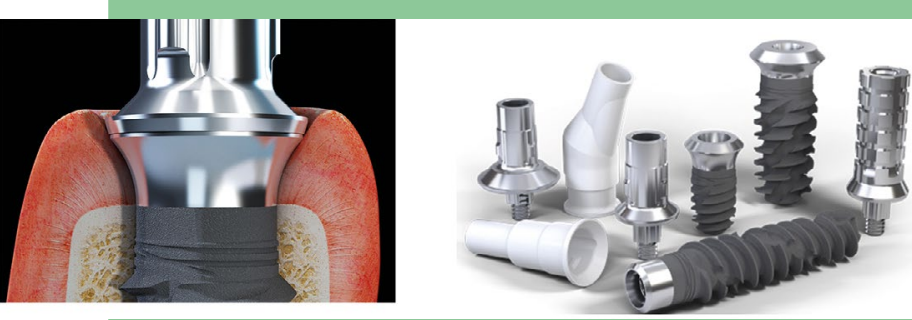

seals the prosthetic on the implant shoulder and is supported by six precision engagement positions - resulting in a restoration with ultimate stability.

What's more, the endosteal design is optimised for primary stability and developed to meet the needs of all treatment protocols. As well as this, Straumann has reduced the invasiveness of this system by using less metal. This means more bone is preserved, ultimately leading to an implant that is incredibly stable and highly likely to enable a smooth healing process. For more details visit http://www. straumann-uk.co/tlx.

\section{New cordless Power Flosser delivers 'The X Factor'}

Philips has announced the launch of a new powered cordless Power Flosser, which is designed to help patients to achieve an effective, consistent and convenient flossing routine, and so improve their gum health in just two weeks. ${ }^{1}$ Launching in March the new cordless Power Flosser builds on the company's air and micro-droplet device know-how, with the introduction of patented 'burst' function technology to remove $99.9 \%$ of plaque in treated areas, to achieve an effortlessly thorough and superior clean.

Meanwhile, its unique cross-shaped nozzle creates four wide streams of water, ensuring it cleans eight times more area than a competitor irrigation appliance with its classic single-stream configuration. This ensures that it is less technique sensitive and can clean the whole mouth in 60-90 seconds. It is this patented 'X-shaped' QuadStream

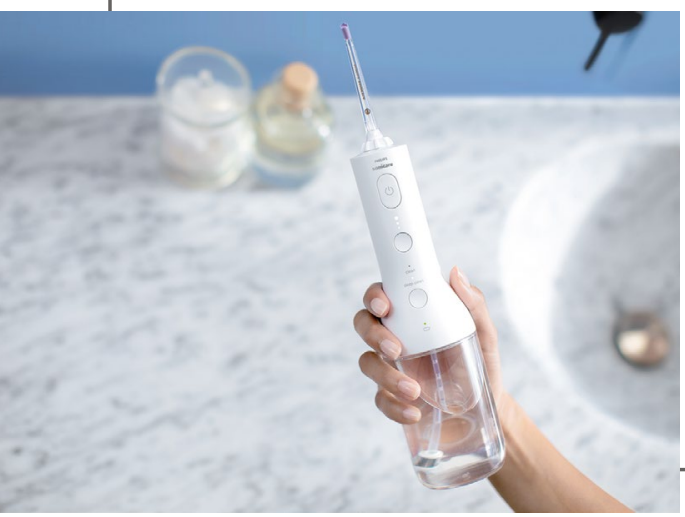

functionality which achieves maximum cleaning coverage by producing a plaquesheering cleaning action, and combines it with a centre jet for superior clean interproximally and along the gumline. Whilst the designers have considered its ergonomics and created an extralarge water reservoir means that the recommended 60 second clean can be carried out without refilling the device.

The cordless Power Flosser has 18 different water streams options including three intensities, two modes and two nozzle types which allows for hyperpersonalisation. In addition, newly developed pulse wave technology guides users from tooth to tooth, ensuring patients who struggle with regular flossing won't miss a spot and clean the whole mouth effectively as brief pauses in the cleaning pulsations prompt them to glide the nozzle to the next interproximal space. Meanwhile the 360 degree nozzle rotation ensures frustrated flossers can access hard-to-reach areas which have been off limits to those with manual dexterity issues. The nozzles are recyclable with Terracycle through the Philips dental care recycling programme.

The Sonicare Cordless Power Flosser will be available for sale in dental practices from March 2022. For more information visit: www.philips.co.uk/dentalprofessional.

\section{Seamless workflows}

Sterilisation can be seamless with SciCan's NEW STATIM B vacuum autoclave.

Its enhanced digital capabilities make it entirely user friendly; dry-wrapped loads delivered in 27 minutes!

STATIM B has a 6-litre capacity, yet it is compact, making it the ideal addition for any-size practice. Designed to support infection control of the highest standard, it offers flexible loading, with an easyopening design.

Practise the art of sterilisation with the STATIM B, available from COLTENE.

With a reputation for superior customer service, COLTENE wants to make dentists' lives easier, while upgrading every element of patient care.

For details on the STATIM ${ }^{\circ}$ B, visit https://www.scican.com/eu/products/ autoclaves/statim-b/.

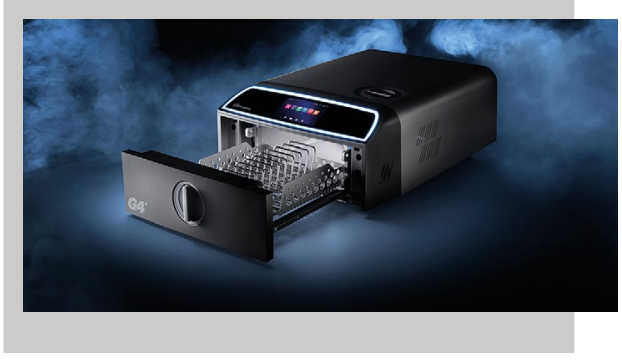

\section{For all your restorative}

\section{needs}

Are you looking for restorative products that can make your life more simple?

$3 \mathrm{M}$ Oral Care has a vast array of restorative solutions available to ensure that you can more easily achieve the results you require. Each of $3 \mathrm{M}$ 's restorative products is the end result of intensive research, meaning that they all have unique features that help you to achieve reliable, long-lasting and aesthetic restorations.

From the Filtek restorative range to Ketac Glass Ionomer from 3M, there's a solution for every indication and style of work.

To find out more call 08705360036 or visit www.3M.co.uk/Dental.

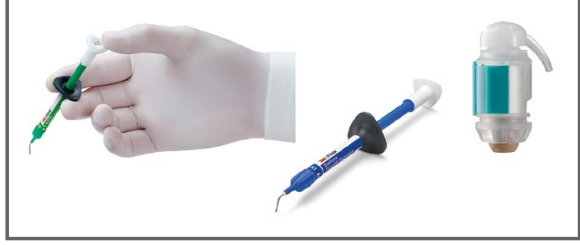

